\title{
The special implications of sepsis
}

Keywords: infections, resistance, staphylococcus, bacteremia, diagnoses

\begin{abstract}
Abbreviations: CDC, center for disease control; ED, emergency department; ORSA, oxacillin resistance among staphylococcus aureus; VRE, vancomycin-resistant enterococci; PRSP, penicillinresistant streptococcus pneumoniae
\end{abstract}

\section{Introduction}

Sepsis is a challenge to the patient and the health care provider because of the circumstances leading to its emergence and the rapidity of development. It may occur in the neonatal or childhood period, in the elderly, postoperatively, or secondary to a localized organ infection or to cancer. The occurrence of sepsis is common and after a decline due to antibiotic developments, its occurrence is increasing with the development of antibiotic resistance. Its diagnosis is characterized by the occurrence of fever and tachycardia with leukocytosis, with increased neutrophil precursors. Unchecked it results in a rapid progression to overwhelm the immune response. This discussion will consider the development of sepsis and special circumstances requiring rapid diagnosis and treatment.

\section{The challenge to the provider}

Two million patients infected annually in the United States alone and at least 23,000 deaths are directly related to infection with antibiotic resistant organisms. ${ }^{1}$ Therefore, it is prudent for the provider to to optimize antibiotic usage (selection, dose, and duration). The result of such efforts might be expected to produce an optimal clinical response, reduce health care costs, mitigate adverse outcomes, and prevent the further development of resistant organisms. The Center for Disease Control (CDC) proposed a National Program to stem the tide of antibiotic resistance: ${ }^{1}$

i. Promoting adherence to appropriate prescribing guidelines among providers.

ii. Decreasing demand for antibiotics for viral upper respiratory infections among healthy adults and parents of young children.

iii. Increasing adherence to prescribed antibiotics for upper respiratory infections.

The emergency department (ED) and surgical facilities as well as urgent care and home care facilities are equally bound to follow these guidelines with specific recommendations. ${ }^{1}$ Epidemiological data indicates that lung infection is the most common infection that is found in sepsis patients. K Pneumoniae, E Coli and S Hominis are the most widely isolated organisms that were found in these patients. The high use of antibiotics with high levels of resistance such as levofloxacin, ceftazidime, ciprofloxacin, cefotaxime, ceftriaxone, and erythromycin requires a policy to control the use of antibiotics to reduce mortality and morbidity in these patients. ${ }^{2}$ A study assessed the antibiotic resistance profiles of 95 isolates of enteric bacteria obtained from wastewater in septic tanks within Chuka University in Kenya. ${ }^{3}$ Isolates were E Coli, Salmonella, Proteus and lebsiella. Resistance to Penicillins was increased and resistance to tetracycline and
Volume 6 Issue I - 2018

\author{
Larry H Bernstein \\ Triplex Medical Diagnostics, USA
}

Correspondence: Larry H Bernstein, Triplex Medical Diagnostics, 54 Firethorn Lane Northampton, MA 01060, USA Email larry.bernstein@gmail.com

Received: December 08, 2017 | Published: February 05, 2018

cephalosporin decreased. Multiple drug resistance was recorded in all strains with E.coli being the most resistant to the antibiotics, followed by Salmonella, Klebsiella and Proteus. While drug resistance in E.coli was significantly different from the other strains, drug resistance between Salmonella and Klebsiella strains was not significantly different. The study helps in identifying changing antibiotic resistance trends in enteric bacteria from sewage effluent necessary to design improved sewage treatment strategies.

\section{Septic joint, urinary infections and pneumonia in the elderly}

Staphylococcus aureus is the most common pathogen causing septic arthritis in orthopedic patients. Those patients infected with MRSA were older, had more chronic medical conditions, and higher values of inflammatory markers. MRSA septic arthritis was also associated with more complications, longer duration of antibiotics, and increased mortality. ${ }^{4}$ Evidence-based guidelines have recently been published to assist in the diagnosis and management of suspected and confirmed septic arthritis. ${ }^{5}$ All suspected septic joints should be aspirated and the synovial fluid examined by microscopy for the presence of crystals and microorganisms. The emergence of multidrug resistant pathogens has led to a search for alternative antimicrobial agents such as linezolid.

First among infection-related causes of death in older persons is pneumonia, but urinary tract infection is the most common bacterial infection seen in geriatric patients. ${ }^{6}$ Other infections in older people include intra-abdominal sepsis, bacterial meningitis, infective endocarditis, infected pressure ulcers, septic arthritis, tuberculosis, and herpes zoster. Antibiotics, vaccination, antisepsis, public health measures, and public sanitation were measures that increased life expectance over the past 50years to approximately 75 in the United States, with older persons accounting for $13 \%$ of the total US population, and infections are no longer among the top three major causes of death. But infectious diseases have re-emerged as important causes of death, disability, and functional decline in the elderly, with bacteremia the ninth cause of death. Older persons have decreased cell-mediated immunity and suffer more chronic diseases, some of which (e.g., cancers, connective tissue diseases) affect the integrity of host resistance. Older patients with pneumonia in the community and in a long-term care setting may have gram-negative bacilli as one of the causes of their lung infection. E. coli accounts for approximately 
$60 \%$ to $70 \%$ of cases of UTI in older adults, with other gram-negative bacilli and enterococci often being isolated, which are treated with broad spectrum antibiotics that will promote colonization of resistant organisms. ${ }^{7}$ This is a major concern in long term care facilities because the patients are transferred from because of transfer to the LTCF of patients who are colonized or infected with such pathogens at other institutions. The most frequent organisms identified are the most frequently isolated resistant pathogens in the older population have been penicillin-resistant Streptococcus pneumoniae (PRSP), methicillin-resistant Staphylococcus aureus (MRSA), vancomycinresistant enterococci (VRE), and multiple-drug-resistant gramnegative bacilli (MDRGNB). Indeed, with essentially no effective antimicrobial agents against some of these pathogens, we would be returning to the "pre-antibiotic era". Reports from different patient populations indicate that multidrug resistance is common among viridans group, oxacillin resistance among Staphylococcus aureus (ORSA) in acute care facilities, and ORSA in community-acquired infection. In addition, Staphylococcus aureus is the most common pathogen causing septic arthritis.

\section{Early therapy}

Sepsis, associated with the systemic inflammatory response (SIRS), has a mortality that ranges between $25 \%$ to $30 \%$ for severe sepsis and $40 \%$ to $70 \%$ for septic shock. ${ }^{8}$ Early goal-directed therapy completed within the first six hours of sepsis recognition is essential for reducing mortality, which requires antibiotic therapy given within one hour of suspected sepsis. ${ }^{89}$ Episodes of multidrug resistant (MDR) gram negative bacteria (GNB) bacteremia were compared with a non-MDR GNB bacteremia group in an 8-year cohort study. MDR GNB accounted for $18.6 \%$ of all neonatal GNB bacteremia in the NICU, especially in those with previous broad-spectrum antibiotic therapy and underlying renal disease. The most frequent mechanism of resistance was extended-spectrum b-lactamase (ESBL) production, which was also associated with infectious complications, and independently associated with a higher overall case-fatality rate. ${ }^{10}$

Cerra and others investigated forty-six patients with surgical sepsis until death or survival to evaluate the effect of exogenous metabolic support on the observed plasma substrate levels and on the differential endogenous utilization of branch chain amino acids. ${ }^{11}$ The administered amino acid load had little effect on substrate levels in patients who died; but significantly affected the observed levels of glycine, isoleucine, and methionine in patients who survived. They concluded that fatal sepsis is associated with an increased release of endogenous valine and isoleucine into plasma, as well as increased plasma levels of tyrosine, proline, and methionine. Furthermore, increased muscle protein catabolism is occurring with a differential utilization of branch chain amino acids and increased use of leucine and isoleucine and reduced use of valine. This auto cannibalism of muscle mass is associated with an increased plasma alanine, but is not influenced by administered amino acid support in the absence of control of the septic process. Transthyretin (TTR) is easily followed in the underweight and the high risk populations in an ambulatory setting, which has a significant background risk of chronic diseases. It is decreased in the systemic inflammatory response syndrome (SIRS. There are a number of physiologic changes associated with SIRS and the injury/repair process that affect TTR. A much better understanding of the significance of this association has emerged from studies of nitrogen and sulfur in health and disease. ${ }^{12,13}$ The most important point is that The systemic inflammatory response syndrome (SIRS) that is an acute response to trauma, burn, or infectious injury characterized by fever, hemodynamic and respiratory changes, and metabolic changes, not all of which are consistently present involves hormonally driven changes in liver glycogen reserves, triggering of lipolysis, lean body proteolysis, and reprioritization of hepatic protein synthesis with up-regulation of synthesis of acute phase proteins and down-regulation of albumin and important circulating transport proteins. The SIRS reaction unabated leads to a recurring cycle with hemodynamic collapse.

\section{Rapid evaluation and results}

A retrospective study analyzed the effects on antibiotic use in patients with severe sepsis and septic shock after implementation of a procalcitonin (PCT)-protocol between 2005 and 2009 with observation of 141 sepsis and organ dysfunction patients who were treated accordingly to a PCT-guided algorithm. ${ }^{14}$ The primary outcome parameters were: antibiotic days on ICU, ICU re-infection rate, 28-day mortality rate, length of stay (LOS) in ICU, mean antibiotic costs (per patient) and ventilation hours. The duration of antibiotic therapy was reduced by an average of 1.0day per year from $14.3 \pm 1.2$ to $9.0 \pm 1.7$ days in $2009(\mathrm{p}=0.02)$. ICU re-infection rate was decreased by yearly $35.1 \%(95 \% \mathrm{CI}-53$ to $-8.5 ; \mathrm{p}=0.014)$ just as ventilation hours by 42 hours per year ( $95 \%$ CI-72.6 to -11.4 ; $\mathrm{p}=0.008)$. ICU-LOS was reduced by 2.7 days per year $(\mathrm{p}<0.001)$. Gil David et al. ${ }^{15}$ analyzed a database for a study of 22,000 laboratory hemograms generated by two Beckman-Coulter Gen-S analyzers over a two month period in a 630 bed acute care facility in Brooklyn. All control samples, patient identifiers, and patients under 23years old were stripped from the dataset. An experienced medical practitioner reviewed all of the data used in generating the algorithm described. The differential diagnoses were outlined prior to beginning the study. An algorithm was developed that characterizes each patient profile and builds a differential metric to identify similar patients who are mapped into a classification. The algorithm successfully classified patients into the diagnosis that were sufficient in sample size, and others are still under observation. The algorithm correctly classified the patients as follows: Microcytic Anemia-99.63\%, Normocytic Anemia-98.03\%, Mild SIRS-73.42\%, Thrombocytopenia-99.52\%, Leukocytopenia-84.83\%, Moderate/Severe SIRS-96.69\% and Normal-93.18\%. The results of the study may have implications for the discussion of rapid identification of sepsis.

\section{Acknowledgements}

None.

\section{Conflict of interest}

The author declares no conflict of interest.

\section{References}

1. Common Sense. The newsletter of the American academy of emergency medicine. 2014;2(1):31-34.

2. Pradipta IS, Sodik DC, Lestari K, et al. Antibiotic resistance in sepsis patients: evaluation and recommendation of antibiotic use. $N \mathrm{Am} \mathrm{J} \mathrm{Med}$ Sci. 2013;5(6):344-352.

3. Mutuku C. Antibiotic resistance profiles among enteric bacteria isolated from wastewater in septic tanks. Amer Sci Res J Eng. 2017;27(1):99-107. 
4. Combs K, Cox K. Clinical outcomes involving patients that develop septic arthritis with methicillin sensitive staphylococcus aureus versus methicillin resistant staphylococcus aureus. J Orthop. 2017;15(1):9-12.

5. Mathews CJ, Coakley G. Septic arthritis: current diagnostic and therapeutic algorithm. Curr Opin Rheumatol. 2008;(4):457-462.

6. Yoshikawa TT. Antimicrobial resistance and aging: beginning of the end of the antibiotic era? J Am Geriatr Soc. 2002;50(7 Suppl):S226-229.

7. Baddour LM, Wilson WR, Bayer AS. Infective endocarditis. Circulation. 2005;111:e394-433.

8. Gauer RL. Early recognition and management of sepsis in adults: the first six hours. Amer Fam Phy. 2013;88(1):44-53.

9. Salomão R, Diament D, Rigatto $\mathrm{O}$, et al. Guidelines for the treatment of severe sepsis and septic shock: management of the infectious agent, source control and antimicrobial treatment. Rev Bras Ter Intensiva. 2011;23(2):145-157.
10. Patolia S, Abate $\mathrm{G}$, Patel $\mathrm{N}$, et al. Risk factors and outcomes for multidrug-resistant Gram-negative bacilli bacteremia. Ther Adv Infect Dis. 2007;5(1):11-18.

11. Cerra FB, Siegel JH, Coleman B, et al. Septic auto cannibalism. A failure of exogenous nutritional support. Ann Surg. 1980;192(4):570-580.

12. Bernstein LH. The systemic inflammatory response syndrome C-reactive protein and transthyretin conundrum. Clin Chem Lab Med. 2007;45(11).

13. Bernstein LH. The Transthyretin inflammatory state conundrum. Current Nutrition \& Food Science. 2012;8(1).

14. Hohn A, Schroeder S, Gehrt A, et al. Procalcitonin-guided algorithm to reduce length of antibiotic therapy in patients with severe sepsis and septic shock. BMC Infectious Diseases. 2013;13:158.

15. David G, Bernstein L, Coifman RR. Generating evidence based interpretation of hematology screens via anomaly characterization. Open Clinical Chemistry Journal. 2011;4:10-16. 